\title{
Utilización de los servicios de salud: una revisión sistemática sobre los factores relacionados
}

\author{
Health services utilization: \\ a systematic review of related factors
}

Raúl Mendoza-Sassi 1,2

Jorge Umberto Béria 2,3

\footnotetext{
1 Departamento de Medicina Interna, Fundação Universidade Federal do Rio Grande. Rua Engenheiro Alfredo Huch 475, Rio Grande, RS 96201-900, Brasil. rams@iname.com 2 Pós-Graduação em Epidemiologia, Departamento de Medicina Social, Faculdade de Medicina, Universidade Federal de Pelotas. Av. Duque de Caxias 250 Pel otas, RS 96030-002, Brasil. jberia@zaz.com.br

3 Pós-Graduação em Saúde Coletiva, Universidade Luterana do Brasil. Rua Miguel Tostes 101, Canoas RS 92420-280, Brasil.
}

A bstract Health services utilization is determined by various factors. In order to study which factors are most important in different countries, a systematic review was conducted from 1970 to 1999. The mean number of visits, proportion of persons who see a doctor, and proportion that concentrate the most visits were similar. Children, chi ldbearing-age women, and the el derly use health care services the most. Lower-income and less educated groups areal so significantly associated with more frequent utilization. Increased heal th needs mediate more frequent utilization by these groups. However, the poorest groups may not receive adequate care, depending on the re spective type of health system. Health need is one of the most important determinants in utilization, and if a health system's equi ty is to be analyzed, one must consider patterns of utilization among social groups in relation to the level of greatest need. Regularly visiting the same physician, a characteristic of accessibility to heal th care services, can determine more adequate utilization. This factor can reduce differences in health care among groups. The authors conclude by proposing a hierarchy of related factors.

Key words Health Services Accessi bility; Medical Care; Delivery of Health Care

Resumen La utilización de los servicios desalud está determinada por diversos factores. Con la fi nalidad de estudiar cuales son los factores más i mportantes y consistentes, fue real izada una revisión si stemática entre los años 1970 y 1999. Se encontró que la media de consultas, la proporción de personas que consultan y la proporción que concentra el mayor número deconsultas fueron similares. Entre los factores demográficos, niños, mujeres en edad fértil y ancianos utilizan más los servicios. Entre los socioeconómicos, la clase social baja y el grupo con menos educación se asociaron con la utilización. El mayor uso está mediado por una mayor necesidad en salud. Pero, según del ti po de sistema, estos grupos menos favorecidos pueden recibir una insuficiente atención. La necesidad en salud es uno de los factores más importantes y, si se desea analizar la equidad del sistema, es necesario tener en cuenta el padrón de utilización entre los grupos sociales para el nivel de mayor necesi dad en salud. Entre los factores rel acionados a los servicios de salud, tener un médico definido determina una utilización más adecuada. Esto puede servir para atenuar las diferencias en la atención médica entre los diferentes grupos sociales. Finalmente, se propone una jerarquía para los factores relacionados.

Palabras clave Accessibilidad a los Servicios de Salud; Atención Médica; Prestación de Atención deSalud 


\section{Introducción}

Los motivos por los cuales una persona consulta van más allá de la carga de morbilidad que pueda estar sufriendo. Es bien conocido el fenómeno del "iceberg de la enfermedad" descrito por Last (1963), donde una pequeña parte de las personas con síntomas consultan con médico. También se sabe que no todos los problemas de salud que tiene una persona son informados al médico en el momento de la consulta, conforme constataron Banks et al. (1975). Por otro lado, existe el gran utilizador responsable por el uso de una gran proporción de las consultas ofrecidas a la población y que, en algunas ocasiones, utiliza los servicios de salud sin que tenga, del punto de vista médico, una justificativa clara. Estos ejemplos son los extremos que pueden ser observados en los padrones de utilización y que son determinados por la necesidad sentida en salud y por otras características.

Al medir la utilización de los servicios, se está estudiando la accesibilidad a los mismos. No es una medida exacta, pero es la más usada en la mayoría de los estudios. Y, al estudiar la utilización-accesibilidad por grupo socioeconómico, también se está eval uando la equidad del sistema en cuestión. Así, este trabajo pretende abordar, mediante la revisión de artículos publicados sobre el tema, cuales son los factores que afectan la util ización de los servicios de salud, cual es el efecto que tienen y cuales pueden ser considerados barreras o, por el contrario, facilitadores del acceso.

Una dificultad en un tema como éste son las limitaciones inherentes al campo de estudio y al objeto de estudio (Novaes, 1996). Además, por tratarse de una revisión que incluye estudios en diversos países, el contexto donde se desarrolla el estudio - representado, entre otras cosas, por el sistema de salud vigente en el país- también contribuye en la complejidad del tema. De cualquier forma, en la tentativa de encontrar factores determinantes de la utilización y no apenas delimitar la revisión a un determinado país, se prefirió realizar una revisión extensiva a diversos países. Si bien esto introduce un elemento de heterogeneidad, las diferencias encontradas en los efectos de un factor sobre la utilización podrán evidenciar la influencia del sistema en la utilización de los servicios de salud.

\section{Metodología}

Para realizar esta revisión sistemática, fueron utilizados los siguientes criterios de selección deartículos: a) Tipo de estudio: estudios con diseño transversal, longitudinal, o caso-control, de base poblacional o de servicios de salud, realizados mediante muestreo al eatorio.

b) Unidad de anál isis: personas de todas las edades.

c) Evento: consultas o internaciones medidas como variable discreta o dicotómica, ocurridas en el período máximo de un año. No fueron seleccionados artículos que se ocupasen de la utilización de servicios por enfermedades específicas.

La búsqueda de artículos fue realizada en el Medline y en los bancos de datos de Bireme ( $\mathrm{Li}$ lacs, AdSaúde e Medcarib), entre 1970 y 1999. Las palabras clave utilizadas para la búsqueda electrónica (en la forma de descriptores o de palabras) fueron: health services, office visits, ambulatory care, primary health care, family practice, family health, cruzadas con health services accessi bility, accessi bility. Se utilizaron también los cal ificadores statistics \& numerical data y utilization. El número de artículos se amplió mediante la identificación de estudios citados en revisiones, en referencias citadas en los artícul os y a través de contacto con autores.

Fue creado un banco de datos en el programa Access, donde se ingresaron los artículos seleccionados. Para cada artículo, fueron extraídos: el año del estudio, el año de publicación, el número de observaciones, el tipo de diseño utilizado, el método estadístico utilizado en el análisis, las variables dependiente e indepen dientes estudiadas, las variables utilizadas para ajuste, la prevalencia de consultas, Ia proporción de la población que consultó y los efectos de los factores estudiados. Finalmente, se construyeron tablas de sistematización con los campos antes señalados, lo que permitió posteriormente un análisis comparativo entre los diversos estudios. A partir de las variables identificadas, se definieron categorías analíticas en donde fueron agru padas las diferentes variables. Las categorías definidas fueron: socioeconómica, demográfica, familiares, soporte social, necesidad en salud y características de los servicios de salud.

\section{Resultados}

\section{Cobertura y promedio de consultas}

Los primeros aspectos estudiados en la revisión fueron algunas características generales sobre la utilización como la cobertura poblacional en un año, el promedio de consultas por habitante/año y la proporción de población 
que concentra mayor número de consultas. En el Tabla 1, se puede observar que la cobertura de consultas en un año es muy semejante entre los países estudiados, situándose en tre el 60 y el 70 por ciento.

En el Tabla 2, aparece el promedio de consultas por habitante y por año. Nuevamente, hay que destacar la similitud entre los datos. El número de consultas para los diferentes países donde se realizaron los estudios se sitúa entre 3 y 4 consultas por persona/año.

También es interesante hacer notar que el fenómeno de la concentración de consultas en un determinado grupo de personas tiene un padrón bastante homogéneo entre diversos países. En el Tabla 3, puede observarse que entre el 10 y el $15 \%$ de las personas utilizan entre un 40 a un $50 \%$ de todas las consultas disponibles. Estas personas son los Ilamados hiperutilizadores, y los motivos que los Ilevan a este comportamiento son diversos. Demers (1995) encontró que este grupo presentaba una mayor cantidad de problemas sicosomáticos y síntomas mal definidos. Otros factores envueltos son la mayor carga de morbidez y la mayor edad.

\section{Factores demográficos}

\section{Edad}

Los grupos extremos (niños y ancianos) utilizan más los servicios que los otros grupos de edad, y esto ocurre independientemente del país en cuestión, como muestran los estudios realizados en los Estados Unidos, por Anderson \& Aday (1978), Parkerson et al. (1995b), Salber et al. (1976), Wan \& Soifer (1974), Wolinski (1978) en Italia, por Mapelli (1993), en Irlanda, por Nolan (1994), en Curazao, por Alberts et al. (1997), en España, por Bellon-Saameno et al . (1995) y en Brasil, por Cesar \& Tanaka (1996) y Costa \& Facchini (1997). Un estudio realizado por Calnan et al . (1994) con adultos, en cuatro países (Inglaterra, Grecia, Rusia y Yugoslavia), también mostró que la utilización de consultas aumentó conforme lo hizo la edad. El efecto es pequeño, como muestran Anderson \& Aday (1978), Bellon-Saameno et al. (1978) y Wolinski (1978). En personas de 50 años o más, después del ajuste con otras variables como necesidad en salud, Costa \& Facchini (1997), en Pelotas, Brasil, observaron que estas personas tenían un riesgo relativo (RR) 10 a 15\% mayor para consultar.

\section{Sexo - género}

Las mujeres utilizan más los servicios, aún después del ajuste con necesidad en salud. Krasnik et al. (1997), en Dinamarca, encontraron que luego de controlar para variables demográficas, socioeconómicas y de necesidad en salud, el sexo femenino se asoció significativamente con la posibilidad de consultar (Odds Ratio = OR 1,76; IC 95\%: 1,42-2,18). En el Brasil, Costa \& Facchini (1997) también encontraron esta asociación (OR 1,84; IC 95\%: 1,73-1,95). En el mismo país, pero en la población de personas con 60 años o más, Pinheiro \& Travassos (1999) vieron que el sexo femenino se asoció positivamente con la utilización de servicios de salud. Además, el uso es mayor en la edad fértil, como muestran los trabajos de Bucket \& Curtis (1986) y de Beland (1988), lo que sugiere una mayor utilización por motivos obstétricos o ginecológicos.

Tamaño - estructura familiar

El tamaño de la familia o el número de hijos también parece influenciar la utilización de consultas, pero los resultados no son consistentes, habiendo inclusive estudios que muestran efectos opuestos. Zastowny et al. (1989), en los Estados Unidos, observaron que personas pertenecientes a familias numerosas consultaron más. Al contrario, Andersen \& Laake

Cobertura de consultas en un año para diversos países.

\begin{tabular}{llll}
\hline Autor & País & Tipo de médico & Cobertura (\%) \\
\hline Salber et al. (1976) & Estados Unidos & Todos & Blancos 70,0/N egros 65,0 \\
Fylkesnes (1993) & Noruega & Médico general & 62,7 \\
Nolan (1994) & Irlanda & Médico general & 60,3 \\
Costa \& Facchini (1997) & Brasil & Todos & 70,0 \\
\hline
\end{tabular}


(1983), en Noruega, encontraron que, después de ajustar para otras variables como necesidad en salud, una familia grande determinó una menor utilización.

La estructura familiar puede ser otro factor que afecta la utilización, pero nuevamente el sentido del efecto depende del país en cuestión. Wolfe (1980) en los Estados Unidos encontró que los niños de familias monoparentales consultaron menos que los otros niños. Ya Balarajan et al . (1992) y Judge \& Benzeval (1993), en Inglaterra, observaron un efecto contrario, siendo que los niños pertenecientes a este tipo de familia consultaron en mayor proporción que los hijos de madres con marido presente en la casa. Si esta variable está asociada con la clase social - como frecuentemente ocurre -, esta diferencia puede estar hablando de la equidad del sistema de salud en el país en cuestión, como veremos más adelante.

\section{Factores socioeconómicos}

Factores como clase social, ingreso, educación, desempleo, etnia o raza, afectan la utilización de los servi cios de salud. Pero la dirección del efecto depende mucho del país en cuestión.

Tabla 2

Promedio de consultas médicas por persona/año para diversos países.

\begin{tabular}{lll}
\hline Autor/Año & País & No. de consultas \\
\hline Salber et al. (1976) & Estados Unidos & Blancos 3,8/Negros 2,8 \\
Mapelli (1993) & Itália & 4,3 \\
Alberts et al. (1997) & Curazao & 3,3 \\
Costa \& Facchini (1997) & Brasil & 3,0
\end{tabular}

Clase social - ingreso

Adler et al. (1993), Freeborn et al. (1977), Judge \& Benzeval (1993), Kaplan et al . (1996) y Yuen et al . (1990), entre otros, mostraron que las clases más bajas tienen una mayor morbilidad y mortalidad. En el Brasil en particular, Travassos et al. (1995) vieron que la morbilidad referida aumentó inversamente al ingreso familiar per capita. Con respecto a la relación entre necesidad y utilización, Andersen \& Aday (1978), Wan \& Soifer (1974) y Fernandez-de-la-Hoz \& Leon (1996), entre otros autores, encontraron que la mayor utilización en estos grupos estaría mediada por una necesidad en salud más acentuada. Así, la relación entre la utilización por estos grupos y los grupos más favorecidos es un indicador de la equidad del sistema. Los estudios muestran que esta relación cambia según el país y el momento del estudio, conforme se verá a seguir.

Balarajan et al. (1987), en Inglaterra, Roos \& Shapiro (1981), en Canadá, y Nolan (1994), en Irlanda, mostraron que la clase más pobre se asoció significativamente con la posibilidad de consultar e internar. Con el desempleo ocurrió la misma situación, y Yuen \& Balarajan (1989), en Inglaterra, encontraron que la categoría desempleada tenía una asociación significativa con la utilización de consultas, aún después de ajustar para tiempo de duración de la enfermedad (OR 1,53; IC 95\%: 1,34-1,76). Esto estaría mostrando la existencia de equidad en la utilización entre los grupos sociales en estos países. Pero el artículo de Haynes (1991), realizado en el mismo país, que muestra que, en la región rural, el grupo sin automóvil tuvo una utilización menor, podría estar indicando la existencia de inaccesibilidad geográfica, al menos para este grupo en particular.

Proporción de la población que utilizó más consultas y proporción del total de consultas que realizó. Diversos países.

\begin{tabular}{llcl}
\hline Autor/Año & País & \% de población & \% del total de consultas \\
\hline Brown et al. (1982) & Canadá & 13 & 46 \\
Kekki \& Laamen (1989) & Finlandia & 14 & 50 \\
Freeborn et al. (1990) & Estados Unidos & 12 & 50 \\
Mapelli (1993) & Itália & 10 & 41 \\
Bellon-Saameno et al. (1995) & España & 15 & 43 \\
\hline
\end{tabular}


Recientemente, Schoen et al. (2000) encontraron que, en Canadá e Inglaterra, Ios indicadores de acceso, entre el grupo con ingreso mayor a la media comparado con el grupo menor, no fueron diferentes. Por el contrario, en Estados Unidos, Australia y Nueva Zelanda, encontraron uso 2 a 3 veces mayor en el grupo demayor ingreso.

La inequidad se puede refl ejar también en la utilización de consultas preventivas. Freeborn et al. (1977), en Ios Estados Unidos, vieron que, entre las mujeres de 40 a 59 años, el grupo de mayor ingreso tuvo una asociación significativa con consultas preventivas. Lo mismo fue observado por Borrell et al. (1999), en España, donde $61 \%$ de las mujeres de la clase social alta realizaron exámenes preventivos para cáncer de cuello de útero, comparados con $32 \%$ de la clase más baja. También el Black Report (Black et al., 1988) describe como, en Gran Bretaña, las mujeres de las clases sociales I y II utilizaron más consultas preventivas (consultas prenatales y dentistas) que las clases inferiores.

Con respecto a las internaciones, Titkow (1983), en Polonia, observó una asociación positiva entre nivel de ingreso e internación. Fernandez-de-la-Hoz \& Leon (1996), en España, observaron que, entre las personas con auto percepción de salud pobre, la categoría de mayor ingreso estaba significativamente asociada con la internación (OR 1,67; IC 95\%: 1,15-2,44). El estudio de Borell et al. (1999), también en España, pero realizado unos años más tarde, no encontró diferencia en el grado de utilización de consultas e internaciones, sugiriendo que los cambios en el sistema de salud de ese país habrían surtido efecto.

Estas diferencias en la utilización por parte de diferentes grupos conducen al papel de las políticas y sistemas de salud en el acceso y la equidad. En China, el estudio de Liu et al. (1999) mostró que la brecha en la utilización de servicios entre grupos de diferente ingreso aumentó entre 1985 y 1993, coincidiendo con las reformas en el sistema. En el Brasil, tres estudios muestran la existencia de inequidad, a pesar de los cambios introducidos. El estudio de Costa \& Facchini (1997), en Pelotas, sur del Brasil, encontró que la clase social baja tuvo $25 \%$ menos de chances de consultar (RR 0,74; IC 95\%: 0,69-0,84). Dela misma forma, el estudio de Pinheiro \& Travassos (1999), en Rio de Janeiro, encontró que el ingreso familiar per capita se asoció positivamente con la utilización de servicios de salud entre la población de 60 años o más. Finalmente, el estudio de Almeida et al. (2000) también encontró que hay evidencias de inequidad en la utilización y el acceso a los ser- vicios, a pesar de las medidas de regulación introducidas.

\section{Educación}

Con respecto a la educación, varios artículos muestran la asociación positiva entre años de estudio y utilización. Salber et al. (1976), en los Estados Unidos, notaron que, cuanto mayor la educación, mayor el número de consultas realizadas. En Inglaterra, Oakley et al. (1993) encontraron que las mujeres con mayor número de años de estudio consultaron después del parto con mayor frecuencia que las mujeres con menor educación. En San Pablo, Brasil, Cesar \& Tanaka (1996) vieron que las personas pertenecientes a jefes de familia con más años de educación consultaron en mayor proporción. También Suarez (1992), en el mismo lugar, vio que, entre los niños menores de un año, el grupo de hijos de madres con mayor escolaridad se asoció significativamente con la mayor utilización de consultas (OR 3,41; IC 95\%: 1,02-11,33). Pero algunos artículos encontraron una asociación inversa entre educación y uso. Es el caso de La Vecchia et al. (1989), en Italia, que encontraron que las personas con mayor estudio tenían un RR de 0,79 (IC 95\%: $0,74-0,85)$ de consultar el médico una vez y un RR de 0,51 (IC 95\%: 0,45-0,58) de consultar dos veces o más, comparados con el grupo de personas con menor educación. Otros, como Pinheiro \& Travassos (1999), no encontraron ninguna asociación en el grupo de 60 años o más.

Ya cuando el resultado analizado son las visitas preventivas, la educación tiene consistentemente un efecto positivo sobre la utilización. Es lo que muestran los artículos de Borras et al. (1999), Borrell et al. (1999), Breen \& Kessler (1994), Calle et al . (1993), Coughlin et al. (1999), Katz \& Hofer (1994) y Zapka et al. (1989). Todos encontraron una asociación significativa entre las dos variables, aún mismo después del ajuste para otros factores.

$\underline{\text { Raza - etnia }}$

Con respecto a la raza, hay evidencias de que pueden haber diferencias en la cantidad y el tipo de prestador de servicio entre grupos raciales. Puede ser estudiada dentro de la categoría demográfica, pero, por su fuerte relación con la categoría socioeconómica, creemos que es más correcto analizarla dentro de esta categoría. Salber et al . (1976), en Estados Unidos, encontraron que, aún después de estratificar por ingreso, la población negra utilizaba más los servicios públicos, en cuanto la población blan- 
ca hacía mayor uso de los servicios privados. Trece años más tarde y en el mismo país, Zastowny et al . (1989) encontraron el mismo resultado. Parkerson et al. (1995a), también en Ios Estados Unidos, observaron que, durante 18 meses de seguimiento, el grupo de mujeres blancas se asoció significativamente con la utilización de seis consultas o más, cuando comparadas con las mujeres negras, pero no controló para ninguna variable socioeconómica. Ya, al contrario, Carr-Hill et al. (1996), en Inglaterra, vieron que, controlando para clase social y desempleo las personas de origen indiana, paquistaní o inmigrantes de Bangladesh, tenían mayor probabilidad de consultar con el médico.

Estas diferencias del efecto de los factores socioeconómicos entre los países estudiados (a veces asociándose con menor utilización, otras veces con mayor utilización) muestran el efecto modificador del sistema de salud vigente. En países con políticas que contemplan la mayor necesidad de los grupos menos favorecidos como es el caso de Inglaterra y Canadá por ejemplo - la equidad se manifiesta por la mayor utilización de los servicios por parte de estos grupos.

Familia y soporte social

La familia también parece influir en la utilización de los servicios de salud. Además del tamaño, ya mencionado antes, otro factor invoIucrado es el stress familiar o la disfunción familiar. Parkerson et al. (1995b), al estudiar el efecto de la utilización de consultas, encontraron que, controlando para edad, sexo y raza las pacientes que realizaban seis visitas médicas o más, tenían mayor stress familiar; el efecto fue significativo, pero muy pequeño (OR 1,02). De la Revilla \& De los Ríos (1994), en España, encontraron que los grandes utilizadores de los servicios de salud (definidos como las personas que utilizaban 9 o más consultas por año) tenían un RR de 6,5 de pertenecer a una familia disfuncional (IC 95\%: 3,95-10,67). Pero el autor no ajustó para otras variables, como edad o sexo, que podrían estar confundiendo. GomezRodriguez et al. (1996), en el mismo país, vieron que pacientes provenientes de un área con situación económica precaria tenían más disfunción familiar y consultaban con mayor frecuencia que los pacientes de otra área con mejor situación socioeconómica. Pero tampoco controlaron para las variables demográficas. Así entonces, el efecto del stressfamiliar sobre la utilización de los servicios de salud puede, en realidad, estar siendo confundido por otras variables no controladas en los estudios realizados.

La madre parece influenciar en la utilización de los servicios de salud a los hijos, aumentando la frecuencia con que consultan, si ellas también son grandes utilizadoras. Esto fue mostrado por Oakley et al. (1993), en Inglaterra, donde hijos recién nacidos de madres caracterizadas como grandes utilizadoras de servicios de salud también fueron grandes utilizadores, y este efecto se mantuvo después del ajuste con las variables socioeconómicas y de necesidad en salud (OR 2,63; IC 95\%: 1,35-5,13).

Con respecto al efecto de la red social sobre el uso de consultas, Krasnik et al. (1997) encontraron que, después de ajustar para factores demográficos y socioeconómicos, la categoría de individuos con menor soporte social (escore de 0 a 3) tuvo una asociación significativa con la menor utilización de los servicios de saIud (OR 0,63; IC 95\%: 0,44-0,91), cuan do comparada con otros grupos.

\section{Características de los Servicios de Salud}

\section{Distancia - recursos existentes}

Algunas característi cas de los servicios de salud pueden afectar la utilización. La distancia entre la casa y el servicio de salud es un factor frecuentemente estudiado por su importancia en el contexto del acceso. Sin embargo, Andersen \& Laake (1983), Carr-Hill et al . (1996), Fylkesnes (1993), Krasnik et al. (1997), Wan \& Soifer (1974) y Costa \& Facchini (1997) no encontraron un efecto significativo. Los estudios fueron realizados en países con una razonable red de atención, lo que puede explicar este resultado. Ya otros países con áreas montañosas o topografía difícil pueden presentar dificultades en la accesibilidad física a los servicios de salud, como es el caso de al gunas regiones de Bolivia (Perry \& Gesler, 2000). También puede ocurrir esta asociación cuando se estudian determinados grupos de población que pueden ser particularmente afectados por la distancia, como en el caso de las personas de edad (Nemet \& Bailey, 2000).

El número de médicos por habitante es otra variable usada para estudiar las características de los servicios de salud en lo que ser refiere a la disponibilidad de recursos. De los artículos de Andersen \& Aday (1978), Fylkesnes (1993) y Mapelli (1993), solamente este último mostró una asociación significativa, talvez por haber incluido la zona rural, donde generalmente la proporción de médicos por habitante es menor. 
Seguro de salud

La variable seguro de sal ud está muy relacionada con la categoría socioeconómica - principalmente cuando es voluntario - , una vez que tiene fuerte relación con el ingreso y la clase social. Pero también puede ser considerada como una característica del financiamiento de los servicios de salud y ser incluido dentro de esta categoría.

El efecto que tiene el factor seguro de salud sobre la utilización es consistente, y la utilización es mayor entre las personas que tienen esta característica. Andersen \& Aday (1978), Kronenfeld (1978) y Wan \& Soifer (1974), en los Estados Unidos, encontraron que, ajustando para variables demográficas, socioeconómicas y de necesidad en salud, las personas con seguro de salud consultaban con mayor frecuencia. Más recientemente, Rosenbach et al. (1999) encontraron que niños con seguro tenían más chances de visitas con el médico y menos chances de visitas en la emergencia. En España, Borras et al. (1999) vieron que las mujeres con seguro de salud privado tenían mayor probabilidad de realizar prevención del cáncer de cuello uterino, aún después de ajustar para educación. Así, el factor seguro de salud no solamente incrementaría el uso de consultas en general, como también determinaría una mayor utilización de los servicios preventivos.

\section{Médico definido}

El hecho de tener un médico definido o un local de consulta definido está asociado también con una mayor utilización. Los investigadores que utilizaron esta variable, como Alpert et al. (1970), Andersen \& Aday (1978), Becker et al . (1974b), Dietrich \& Marton (1982) y Hayward et al. (1991), encontraron que éste fue uno de los factores más importantes en la determinación de la utilización. El tener un médico definido también puede ser considerado como un elemento de la calidad de la atención, vinculado a la continuidad de la asistencia y de la relación médico paciente, conforme explica Donabedian (1984).

La prevalencia de médico definido, en los Estados Unidos, se sitúa entre 70 y $80 \%$, como muestran los trabajos de Hayward et al. (1991), Lambrew et al. (1996), Lave et al. (1979) y Mainous \& Gill (1998). En el Brasil, el estudio de Stein (1998) mostró que un 30\% de los pacientes entrevistados referían tener un médico definido. Este estudio fue realizado en el sector de emergencia de un hospital de Porto Alegre, sur del Brasil, y que mantiene un programa de médico de familia en su área de influencia. Estas dos características pueden haber afectado de formas diferentes la prevalencia encontrada. Por un lado, parte de la población que recurre a una emergencia lo hace por no tener una fuente de cuidados médicos definida, como se verá más adelante. Por otro lado, al realizarse el estudio en un hospital que tiene un programa de médico de familia en su área de influencia - cuya característica es justamente el médico definido -, muchos de los pacientes que fueron estudiados podrían pertenecer a este programa. Así la medida de frecuencia de este factor podría estar modificada, en cualquier sentido.

El factor médico definido afecta tanto el número de consultas médicas, como de emergencia o las internaciones. Stein encontró que, después de ajustar para variables como edad, tiempo de traslado al servicio, consultas anteriores e hipertensión arterial, el grupo de pacientes con esta característica se asoció significativamente con la utilización del servicio de emergencia por motivos realmente urgentes (OR 2,63; IC 95\%: 1,59-4,34) y con la utilización de servicios de atención primaria (OR 3,58; IC 95\%: 2,19-5,87). Wasson et al. (1984), en los Estados Unidos, vieron que personas con 55 añoso más que recibieron cuidados del mismo médico durante 18 meses de seguimiento tuvieron menor número de internaciones de emergencia y menor cantidad de días de internación. Lambrew et al. (1996), en el mismo país, encontraron que, ajustando para edad, sexo, raza, seguro de salud, educación, ingreso, empleo y necesidad en salud, la categoría con médico definido se asoció significativamente con la posibilidad de consultar, cuando comparada con las que no tenían ni médico definido, ni un local definido para consultar (OR 1,97; IC 95\%: 1,58-2,45).

Cuando se estudia separadamente el efecto de médico definido y de local definido, el efecto del primer factor es mayor. En el artículo anterior, Lambrew observó que el grupo que tenía médico definido tuvo una asociación significativa con la posibilidad de consultar, cuando comparado con quién tenía apenas un local definido de consulta (OR 1,47; IC 95\%: 1,30-1,67). Mainous \& Gill (1998) encontraron que, ajustando para otras variables como edad, sexo, raza, local de residencia, morbilidad y número de visitas de ambulatorio, el grupo con médico definido tuvo menor probabilidad de internar que el grupo que tenía un local definido, pero no un médico fijo (OR 0,75; IC 95\%: 0,66-0,87).

Con respecto a la relación entre médico definido y la utilización de los servicios de emergencia, Sox et al. (1998) encontraron que, des- 
pués de ajustar para otras variables (demográficas, socioeconómicas, seguro de salud y número de enfermedades crónicas), la categoría sin médico definido se asoció significativamente con la utilización de los servicios de emergencia (OR 1,8; IC 95\%: 1,4-2,4). Por otro lado, Baker et al. (1994) vieron que las personas que identificaban al servicio de emergencia como su lugar habitual de consulta hicieron menos consultas médicas que aquellas personas que tenían un local definido diferente al servicio de emergencia ( 1,8 consultas vs. 2,4 consultas; $\mathrm{p}<0,001$ ).

Finalmente, sobre la asociación entre médico definido y consultas preventivas, los dos artículos de Becker - Becker et al. (1974a, 1974b) - no mostraron diferencias significativas. Gordis \& Markowitz (1971) y Spivack et al . (1980) tampoco encontraron diferencias. Pero otros estudios sí encontraron una asociación significativa. Alpert et al. (1970) encontraron que niños con médico definido realizaban más consultas preventivas y menos por enfermedad. Kirkman-Lift \& Kronenfeld (1992) vieron que quien tenía una fuente regular de cuidados médicos tenía una mayor probabilidad de realizar una mamografía.

Otros aspectos relacionados con la calidad de la atención parecen estar afectados por la continuidad del cuidado médico, operacionalizado como médico definido. Forrest $\&$ Starfield (1998) encontraron que quien tenía continuidad en la atención presentaba una menor probabilidad de esperar más tiempo por la consulta. De la misma forma, Sox vio que el grupo sin médico definido tuvo una asociación significativa con la variable demora para consultar (OR 1,6; IC 95\%: 1,2-2,1), cuando comparado con el grupo con esta característica.

El factor médico definido está asociado con los factores socioeconómicos, como veremos a seguir. Hayward et al. (1991), en los Estados Unidos, encontraron que, ajustando para otras variables, el grupo más pobre se asoció significativamente con el hecho de no tener un médico definido en razón de barreras económicas (OR 5,2; IC 95\%: 2,6-10,6). También encontraron que la categoría de menor escolaridad se asoció significativamente con la no presencia de un médico fijo (OR 2,5; IC 95\%: 1,3-4,8). Merzel (2000) encontró que, entre los hombres, los factores socioeconómicos fueron predictores para tener una fuente regular de cuidados médicos. Con respecto a la raza, el estudio de Lave et al. (1979), también en los Estados Unidos, observó que, después de ajustar para las variables ingreso y educación, los negros tenían mayor probabilidad de no tener esta característica.
Por todo lo visto hasta ahora, el factor médico definido puede ser consi derado como uno de los factores más importantes en la determinación de la utilización. Las personas con esta característica utilizan de forma más adecuada los servicios de salud, y su presencia puede ser considerada como un marcador de la calidad de la atención.

A parte de las señaladas, otras características pueden ser estudiadas en la categoría servicios de salud. El tiempo en que permanecen abiertos los servicios de salud (accesibilidad temporal), el costo total que la persona tiene para utilizar los servicios de salud, y el costo con medicamentos (accesibilidad económica) son ejemplos de otros factores que afectan la utilización.

\section{Necesidad en salud}

En esta categoría se consideran aquellas variables que reflejan las alteraciones en el estado de salud de la persona y que pueden llevar a consultar. Puede ser medida a través de informaciones médicas directas o indirectas (registros), o ser referida por la persona (morbilidad referida). En este último caso se puede medir por la auto percepción de salud, por el número de síntomas, por el número de enfermedades crónicas padecidas o por el número de días restringidos para las actividades normales debido a la enfermedad, entre otras. En general, los estudios de base poblacional utilizan este tipo de definición de necesidad, y todos los artículos revisados la abordaron desde este punto de vista.

Los estudios de Andersen \& Aday (1978), Antonovsky et al. (1989), Conelly \& Smith (1991), Fylkesnes (1993), Mapelli (1993), Fernandez-Mayoralas et al. (2000) y Wan (1982) encontraron una asociación significativa con la utilización, siendo ésta siempre el factor principal. En el Brasil, el estudio de Pinheiro \& Travassos (1999) mostró que, en el grupo de 60 años o más, la morbilidad referida fue el factor más importante en la explicación de la variación de la utilización de servicios de salud (OR 4,35; p <0,001). Fernandez-de-la-Hoz \& Leon (1996), Parkerson et al. (1995b) y Roos \& Shapiro (1981) encontraron una relación directa y una ten dencia linear entre los diferentes niveles de necesidad (medida por la auto-percepción en salud) y la utilización de servicios. También hay una relación entre la auto-percepción de salud baja y la salud mental, como muestra el estudio de Conelly et al. (1989), donde pacientes con salud pobretenían mayor 
depresión, ansiedad y preocupación por su estado de salud.

Al estudiar la utilización de los diferentes grupos sociales por nivel de necesidad pueden surgir diferencias que podrían de otro modo pasar desapercibidas. Es lo que muestran los trabajos de Kleinman et al. (1981) y de Newacheck (1988). Cuando estudiaban la utilización sin separar por grupo de necesidad, no existían diferencias entre los grupos sociales. Pero, cuando estudiaban el grupo con mayor necesidad en salud, Ias personas de las clases sociales más bajas, consultaban en menor proporción que las personas de la clase alta, manifestando así la existencia de inequidad. Esto resalta la importancia que tiene la medición adecuada de la variable necesidad en salud y el control que debe ser real izado con las variables socioeconómicas. En ciertos artículos, esta mensuración y comparación de la utilización entre grupos conforme el nivel de necesidad es llamada de equidad "horizontal", en contraposición a la equidad (vertical) estudiada por la medida de la utilización entre diferentes grupos sociales, pero sin tener en cuenta el nivel de necesidad.

\section{Satisfacción}

La sati sfacción del usuario es, sin lugar a dudas, otra de las variables que afecta la utilización de los servicios de salud, pero también puede ser estudiada como variable dependiente, porque hay entre las dos variables causalidad reversa. Uno de los artículos que aborda la cuestión desde este último punto de vista sugiere que la satisfacción depende del contexto del proveedor de servicios y de las características individuales, habiendo por tanto mucha variabilidad (Zastowny et al., 1989). Los autores encontraron una asociación inversa entre utilización y satisfacción en el grupo de raza blanca. Esto puede significar que las personas que no quedan satisfechas con la atención deben consultar nuevamente, aumentando, por lo tanto, el número de consultas realizadas en este grupo. Al contrario, quien queda satisfecho con la atención recibida no necesita volver a visitar médico, lo que disminuye el número de consultas en este grupo.

En el Tabla 4, se presentan algunos de los factores asociados a la utilización de servicios y a sus respectivas medidas de efecto, identificados a partir de los artículos revisados.

\section{Algunas consideraciones sobre las relaciones jerárquicas entre las diferentes categorías}

Si representásemos las diferentes categorías estudiadas según su localización jerárquica con respecto a la variable dependiente (utilización de los servicios de salud), tendríamos lo que se observa en la Figura 1. Las categorías

Algunos factores asociados a la utilización de servicios, según país y medidas de efecto.

\begin{tabular}{|c|c|c|c|c|}
\hline Autor/Año & País & Tipo de utilización & Factor & $\begin{array}{l}\text { Medida del efecto } 1 \\
\text { OR (IC } 95 \%)\end{array}$ \\
\hline Krasnik et al. (1997) & Dinamarca & Consultas & Sexo femenino & $1,76(1,42-2,18)$ \\
\hline Costa \& Facchini (1997) & Brasil & Consultas & Sexo femenino & $1,84(1,73-1,95)$ \\
\hline Costa \& Facchini (1997) & Brasil & Consultas & Clase social baja & $0,74(0,69-0,84)^{2}$ \\
\hline Borrell et al. (1999) & España & Sin visita preventiva & Clase social baja & $4,74(1,68-13,41)$ \\
\hline Fernadez-de-la-Hoz \& Leon (1996) & España & Internación & Mayor ingreso & $1,67(1,15-2,44)$ \\
\hline Suarez (1992) & Brasil & Consultas & Mayor educación & $3,41(1,02-11,33)$ \\
\hline La Vecchia et al. (1989) & Italia & Consultas & Mayor educación & $0,79(0,74-0,85)$ \\
\hline Yuen \& Balarajan (1989) & Inglaterra & Consultas & Desempleo & $1,53(1,34-1,76)$ \\
\hline Krasnik et al. (1997) & Dinamarca & Consultas & Muy bajo soporte social & $0,63(0,44-0,91)$ \\
\hline Lambrew et al. (1996) & Estados Unidos & Consultas & Médico definido & $1,97(1,58-2,45)$ \\
\hline Mapelli (1993) & Italia & Media de consultas & Salud pobre/Salud excelente & $7,4 / 2,5^{3}$ \\
\hline Pinheiro \& Travassos (1999) & Brasil & Consultas/Internaciones & Problema de salud & $4,35(p=0,001)$ \\
\hline
\end{tabular}

1 o dds ratio y intervalo de confiança $95 \%$ ajustados para diversas variables, dependiendo del estudio (ver texto).

2 Razón de prevalencias.

3 Médias. 
Figura 1

Relación jerárquica de las diferentes categorías analíticas relacionadas a la utilización de los servicios de salud.

\begin{tabular}{l|l|} 
1er Nível & $\begin{array}{l}\text { Factores } \\
\text { socioeconómicos }\end{array}$ \\
20 Nível & Familia \\
3er Nível & $\begin{array}{l}\text { Comportamiento } \\
\text { en la búsqueda de } \\
\text { cuidados médicos }\end{array}$
\end{tabular}

40 Nível

Variable dependiente
Factores demográficos

Eventos estresantes

\section{Soporte social}

servicios de salud
Necesidades en salud
Satisfacción del usuario

Utilización de servicios

de salud

Satisfacción del usuario más distantes con respecto a la variable dependiente están representadas en la parte superior de la figura. En la medida que se desciende, se pasa a categorías que se relacionan más próximamente con el resultado estudiado. Las categorías localizadas superiormente ejercen sus efectos (totales o parciales) sobre la variable dependiente, directamente o a través de las categorías situadas por debajo de ellas.

La categoría socioeconómica y la categoría factores demográficos estarían en el primer nivel. La categoría socioeconómica (con las variables clase social, nivel de ingreso, educación, entre otras) podría ser mediada total o parcialmente por las variables de los niveles inferiores. Ya la categoría demográfica (edad, sexo, estado civil) muy posiblemente sería mediada a través de la categoría necesidad en salud.

En un segundo nivel, podrían aparecer la categoría familia (con variables como stress fa- miliar, familia extendida y familia monoparental), la categoría red social (con la variable soporte social) y la categoría eventos estresantes. Estas categorías estarían en un nivel jerárquico superior a las categorías del nivel siguiente e inferior (características de los servicios de salud, comportamiento en la búsqueda de cuidados médicos y necesidad en salud) y a través de ellas se relacionarían con la variable dependiente. Ya los eventos estresantes podrían estar relacionados con la variable dependiente a través de una mayor necesidad en salud, una vez que están asociados a los disturbios siquiátricos menores (Lima et al., 1996).

En el tercer nivel, estarían las categorías característica de los servicios de salud, la necesidad en salud y el comportamiento en la búsqueda de cuidados médicos (definida a través de algunas características y conductas que la persona tiene frente a la enfermedad). Esta úl- 
tima variable ha sido muy estudiada en las madres de niños con algunas enfermedades infecciosas comunes (Vos, 1994), y se vio que afecta la utilización.

Finalmente, en el cuarto nivel, se encontraría la variable satisfacción del usuario, una variable que guardaría una relación de causalidad reversa con la variable dependiente y, por este motivo, estaría representada con líneas discontinuas. Cada evento de utilización de servicio de salud generaría una respuesta de satisfacción y, en este caso, la variable estaría en una posición más distante con respecto a la utilización. Lo mismo ocurre con los usuarios que consultarían por la primera vez. Pero también, como es el caso de las personas que ya usaron los servicios de salud, la satisfacción pasada determinaría la utilización presente o futura, por la cual esta categoría pasaría a estar representada en posición superior a la variable dependiente.

\section{Conclusiones}

La cobertura de consultas anuales muestra similitud entre los diversos países estudiados, así como el promedio de consultas por año y la proporción de la población que utiliza más los servicios de salud. Esta homogeneidad indica que, al menos entre los trabajos localizados, hay un padrón de utilización que aparentemente extrapola los límites geográficos de los países. Hay que señalar que los estudios a que se hacen referencia fueron realizados en su mayoría en países que, si bien guardan diferencias con relación a los tipos de sistemas de salud vigentes - mayor o menor grado de accesibilidad y de equidad -, tienen una red de atención más o menos desarrollada.

Entre los factores demográficos, se observó que las edades extremas y el sexo femenino utilizan más los servicios de salud. El efecto es mediado, al menos en parte, por una mayor necesidad en salud. El tamaño y la estructura familiar están asociados a la utilización, pero la dirección del efecto depende del país en estudio. Así, por ejemplo, en países con sistemas de salud de acceso universal, los hijos de madres solteras consultan más. En cambio, en países con sistemas de salud donde hay mayor número de barreras, este mismo grupo consulta en menor proporción, dejando en evidencia la desigualdad.

Los factores socioeconómi cos determinan la utilización de los servicios de salud, principalmente a través de las categorías necesidad en salud y característica de los servicios de sa- lud. Los grupos más pobres, los cuales tienen una mayor carga de enfermedad, tienden a utilizar menos los servicios de salud, si la accesibilidad al sistema no les es favorable. Nuevamente, las diferencias observadas entre grupos de países manifiestan la importancia de las políticas y los sistemas de salud. En países con sistemas donde la accesibilidad es universal, como Inglaterra o Canadá, las personas de clase baja tienen mejor acceso a los servicios. En estos casos, el sistema funciona como un modificador de efecto y promueve la equidad. En otros casos, los sistemas no consiguen disminuir las desigualdades. Es la expresión de la ley de los cuidados inversos de Hart (1971).

Entre las características familiares, personas provenientes de familias disfuncionales realizan más consultas. Pero es necesario realizar nuevos estudios donde se proceda al ajuste con las variables socioeconómicas y demográficas, ya que pueden estar confundiendo los resultados. Sí, hay evidencias claras de que el padrón de utilización de la madre determina el padrón de los hijos, mostrando una vez más la importancia de la figura materna en el cuidado de la salud de los niños.

Entre los factores considerados en la categoría servicios de salud, la distancia al servicio y el mayor número de médicos por habitante aumentan la utilización. En países con una red de servicios adecuada, estos factores pierden importancia. Pero, en estos mismos países, la distancia geográfica puede volver a ser una barrera para grupos muy específicos de personas, como son las personas de edad o aquellas con al gún tipo de limitación funcional.

Las personas que poseen seguro de salud voluntario tienden a utilizar más los servicios de salud. Pero, como esta característica está asociada al mayor ingreso, favorece la utilización apenas en los grupos menos necesitados, al imentando de esta manera la inequidad.

Otro de los otros factores de la categoría servicios de salud es el médico definido. Este factor tiene un importante efecto en la determinación de la utilización. Las personas que tienen esta característica, no sól o utilizan más los servicios de consulta e internan menos veces, como también utilizan los servicios de emergencia, cuando tien en realmente un motivo urgente. Como este factor está asociado, en algunos países, a los grupos sociales de me jores condiciones económicas, la ausencia de médico definido en los grupos socioeconómicos menos favorecidos puede ser considerado un elemento promotor de inequidad.

Otros factores que tendrían importancia en la determinación de la utilización los servicios 
de salud son el comportamiento en la búsqueda de cuidados médicos - que refleja los aspectos de cómo la persona reacciona frente a la enfermedad - y los eventos estresantes, que pueden determinar una mayor utilización en función de una mayor necesidad en salud. Con respecto al primer factor, hay estudios que muestran que las clases más bajas no perciben la necesidad de consultar de la misma forma que las clases más al tas (Fraser, 1992). Habría que considerar medidas de educación específicas para estos grupos que promoviesen cambios en las actitudes frente a la enfermedad.

Finalmente, hay que consi derar la satisfacción del usuario. Esta característica tanto pue-

\section{Referencias}

ADLER, N.; BOYCE, W.; CHESNEY, M .; FOLKMAN, S. \& SYME, L., 1993. Socioeconomic inequalities in health. No easy solution. JAMA, 269:3140-3145.

ALBERTS, J. F.; SANDERMAN, R.; EIMERS, J. M. \&VAN DER HEUVEL, W. J. A., 1997. Socioeconomic inequity in health care: A study of services utilization in Curaçao. Social Science and Medicine, 45:213-220.

ALMEIDA, C.; TRAVASSOS, C.; PORTO, S. \& LABRA, M., 2000. Health sector reform in Brazil: A case study of inequity. International Journal of Health Services, 30:129-162.

ALPERT, J. J.; KOSA, J.; HAGGERTY, R. J.; ROBERTSON, L. S. \& MEAGARTY, M. C., 1970. Attitudes and satisfactions of low-income families receiving comprehensive pediatric care. American Journal of Public Health, 60:499-506.

ANDERSEN, A. S. \& LAAKE, P., 1983. A causal model for physician utilization: Analysis of Norwegian data. Medical Care, 21:266- 278.

ANDERSEN, R. \& ADAY, L. A., 1978. Access to medical care in the U.S.: Realized and potential. Medical Care, 16:533-546.

ANTONOVSKY, H.; MAOZ, B.; PILPEL, D. \& ARAD, T., 1989. Personal and health factors associated with frequency of visits to the primary health clinic. Family Practice, 6:182-187.

BAKER, D. W.; STEVENS, C. D. \& BROOK, R. H., 1994. Regular source of ambulatory care and medical care utilization by patients presenting to a Public Hospital Emergency Department. JAMA, 271: 1909-1912.

BALARAJAN, R.; YUEN, P. \& MACHIN, D., 1987. Socioeconomic differentials in the uptake of medical care in Great Britain. Journal of Epidemiology and Community Health, 41:196-199.

BALARAJAN, R.; YUEN, P. \& MACHIN, D., 1992. Deprivation and the general practitioner workload. BMJ, 304:529-534.

BANKS, M .; BERESFORD, S.; MORRELL, D.; WALLER, J. \&WATKINS, C., 1975. Factors influencing demand of primary medical care in women aged 2044: A preliminary report. International Journal of Epidemiology, 4:189-195. de determinar la utilización - si la persona ya utilizó los servicios de salud -, como ella puede estar determinada por la utilización - si es la primera vez que los utiliza - habiendo por lo tanto una relación de causalidad reversa entre ellas. Además, al contrario de lo que se espera en la relación satisfacción-consumo (a mayor satisfacción, mayor consumo), hay alguna evidencia de que la relación en la utilización de los servicios de salud puede ser inversa. Así, si las personas no consiguen satisfacer sus necesidades en salud, buscan nuevamente consultar con otro prestador de servicios, hasta satisfacerlas.

BECKER, M. H.; DRACHMAN, R. H. \& KIRSCHT, J. P., 1974a. A field experiment to evaluate various outcomes of continuity of physician care. American Journal of Public Health, 64:1062.

BECKER, M. H.; DRACHMAN, R. H. \& KIRSCHT, J. P., 1974b. Continuity of pediatricians: New support for an old shibboleth. Medical Care, 84:599.

BELAND, F., 1988. Utilization of health services as events: An exploratory study. Health Services Research, 23:295-310.

BELLON-SAAMENO, J.; DELGADO-SANCHEZ, A.; LUNA-DEL-CASTILLO, J. D. \& LARDELLI-CLARET, P., 1995. Influence of age and sex on various types of utilization of the primary health care. Gaceta Sanitaria, 9:343-353.

BLACK, D.;TOWNSEND, P.; DAVIDSON, N. \&WHITEHEAD, M., 1988. Inequalities in Health: The Black Report and the Health Divide. London: Penguin Books.

BORRAS, J. M.; GUILLEN, M .; SANCHEZ, V.; JUNCA, S. \&VINCENTE, R., 1999. Educational level, voluntary private health insurance and opportunistic cancer screening among women in Catalonia (Spain). European Journal of Cancer Prevention, 8:427-434.

BORRELL, C.; ROHLFS, I.; FERRANDO, J.; PASARIN, M. I.; DOMINGUEZ-BERJON, F. \& PLASENCIA, A., 1999. Social inequalities in perceived health and the use of health services in a Southern European Urban Area. International Journal of Health Services, 29:743-764.

BREEN, N. \& KESSLER, L., 1994. Changes in the use of screening mammography: Evidence from the 1987 and 1990 national health interview survey. American Journal of Public Health, 84:62-67.

BROWN, G. B.; HUMPHREY, B.; PALLISTER, R.; BROWN, J. A. \& SHETZER, L., 1982. Prevalence and characteristics of frequent attenders in a prepaid Canadian Family Practice. Journal of Family Practice, 14:63-71.

BUCKET, D. \& CURTIS, S., 1986. Socio demographic variation in perceived illness and the use of primary care: The value of community survey data for primary care service planning. Social Science and Medicine, 23:737-744. 
CALLE, E. \& AL, E., 1993. Demographic predictors of mammography and pap smear screening in U.S. women. American Journal of Public Health, 83: 53-60.

CALNAN, M.; WILLIAMS, S.; RAMIC, H. \& PROKHORSKAS, R. O., 1994. Major determinants of consumer satisfaction with primary care in different health systems. Family Practice, 11:468-478.

CARR-HILL, R. A.; RI CE, N. \& ROLAND, M., 1996. Socioeconomic determinants of rates of consultation in general practice based on Fourth National Morbidity Survey of General Practices. BMJ, 312: 1008-1012.

CESAR, C. \& TANAKA, O., 1996. Household Surveys as an evaluation tool for health services: A case study from the Southeast Region of the Greater São Paulo Metropolitan Area, 1989-1990. Cadernos de SaúdePública, 12(Sup. 2):59-70.

CONELLY, J. E.; PHILBRICKK, J. T.; SMITH, J. R. \& KAISER, D. L., 1989. Health perceptions of primary care patients and the influence on health care utilization. Medical Care, 27:S99-S109.

CONELLY, J. E. \& SMITH, G. R., 1991. Healthy patients who perceive poor health and their use of primary care services. Journal of General Internal Medicine, 6:47-51.

COSTA, J. D. \& FACCHINI, L. A., 1997. Utilização de serviços ambulatoriais em Pelotas: Onde a população consulta e com que freqüência. Revista de SaúdePública, 31:360-369.

COUGHLIN, S. S.; UHLER, R. J. \& BLACKMAN, D. K., 1999. Breast and cervical cancer screening practices among American Indian and Alaska native women in the United States, 1992-1997. Preventive Medicine, 29:287-295.

DE LA REVILLA, L. \& DE LOS RIOS, A. M., 1994. The utilization of health services and the motives for consultation as indicators of family dysfunction. Atención Primaria, 13:73-76.

DEMERS, M., 1995. Frequent users of ambulatory health care in Quebec: The case of doctor-shoppers. Canadian Medical Association Journal, 153: 37-42.

DIETRICH , A. J. \& MARTON, K. I., 1982. Does continuous care from a physician make a difference? Journal of Family Practice, 15:929.

DONABEDIAN, A., 1984. La Calidad de la Atención Médica. Definición y Métodos de Evaluación. México, DF: La Prensa Médica Mexicana.

FERNANDEZ-DE-LA-HOZ, K. \& LEON, D. A., 1996. Self-perceived health status and inequalities in use of health services in Spain. International Journal of Epidemiology, 25:593-603.

FERNANDEZ-MAYORALAS, G.; RODRIGUEZ, V. \& ROJO, F., 2000. Health services accessibility among Spanish elderly. Social Science and Medicine, 50: 17-26.

FORREST, C. B. \& STARFIELD, B., 1998. Entry into primary care and continuity: The effects of access. American Journal of Public Health, 88:1330-1336.

FRASER, C., 1992. Setting the scene. In: Clinical Method. A General Practice Approach (R. C. Fraser, ed.), pp. 1-24, Cambridge: Butterworth Heinemann.

FREEBORN, D. K.; POPE, C. R. \& DAVIS, M. A., 1977. Health status, socioeconomic status and utilization of outpatient services for members of a pre- paid group practice. Medical Care, 15:115-128.

FREEBORN, D. K.; POPE, C. R.; MULOLY, J. P. \& MCFARLAND, B. H., 1990. Consistenly high users of medical care among elderly. Medical Care, 28:527-540.

FYLKESNES, K., 1993. Determinants of health care utilization - Visits and referrals. Scandinavian Journal of Social Medicine, 21:40-50.

GOMEZ-RODRIGUEZ, E.; GERVAS, J.; HERNANDEZ, M. M. \& MORENO, R. P., 1996. Socioeconomic status, chronic morbidity and health services utilization by families. Family Practice, 13:382-385.

GORDIS, L. \& MARKOWITZ, M., 1971. Evaluation of the effectiveness of comprehensive and continuous pediatric care: Part I. Effectiveness of comprehensive care in influencing infant health. $\mathrm{Pe}$ diatrics, 48:766.

HART, J., 1971. The inverse care law. Lancet, 1:405-412.

HAYNES, R., 1991. Inequalities in health and health service use: Evidence from the general household survey. Social Scienceand Medicine, 33:361-368.

HAYWARD, R. A.; BERNARD, A. M.; FREEM AN, H. E. $\&$ COREY, C. R., 1991. Regular source of ambulatory care and access to health services. American Journal of Public Health, 81:434-438.

JUDGE, K. \& BENZEVAL, M., 1993. Health in equalities: New concerns about children of single mothers. BMJ, 306:677-680.

KAPLAN, G.; PARNUK, E.; LYNCH, J.; COHEN, R. \& BALFOUR, J., 1996. Inequality in income and mortality in the United States: Analysis of mortality and potential pathways. BMJ, 312:999-1003.

KATZ, S. \& HOFER, T., 1994. Socioeconomic disparities in preventive care persist despite universal coverage. JAMA, 272:530-534.

KEKKI, P. \& LAAMEN, R., 1989. Análisis del uso y cobertura de una población de un distrito de atención en Finlandia. Un enfoque epidemiológico. Atención Primaria, 6:540-546.

KIRKM AN-LIFT, B. \& KRONENFELD, J., 1992. Access to cancer screening services for women. American Journal of Public Health, 82:733-735.

KLEINMAN, J. C.; GOLD, M. \& MAKU C, D., 1981. Use of ambulatory medical care by the poor: Another look at equity. Medical Care, 19:1011-1029.

KRASNIK, A.; SAWITZ, A.; KEIDING, N. \& HANSEN, E., 1997. Determinants of general practice utilization in Denmark. Danish Medical Bulletin, 44: 542-546.

KRONENFELD, J. J., 1978. Providers variables and the utilization of ambulatory care services. Journal of Health and Social Behavior, 19:68-76.

LA VECCHIA, C.; NEGRI, E.; PAGANO, R. \& DECARL, A., 1989. Education, prevalence of disease and frequency of health care utilization: The 1983 Italian National Health Survey. Journal of Epidemiology and Community Health, 41:161-163.

LAMBREW, J. M .; DEFRIESE, G. H.; CAREY, T. S.; RICKETTS, T. C. \& BIDDLE, A. K., 1996. The effects of having a regular doctor on access to primary care. Medical Care, 34:138-151.

LAST, J., 1963. The iceberg: Completing the clinical picture in general practice. Lancet, 2:28-31.

LAVE, J. R.; LAVE, L. B.; LEI NHARDT, S. \& NAGIN, D., 1979. Characteristics of individuals who identify a regular source of medical care. American Journal of Public Health, 69:261-267. 
LIMA, M. S.; BÉRIA, J. U.; TOMASI, E.; CONCEIÇÃO, A. T. \& M ARI , J. J., 1996. Stressful life events and minor psychiatric disorders: An estimate of the population attributable fraction in a Brazilian community-based study. International Journal of Psychiatry in Medicine, 26:211-222.

LIU, Y.; HSIAO, W. C. \& EGGLESTON, K., 1999. Equity in health and health care: The Chinese experience. Social Scienceand Medicine, 49:1349- 1356.

MAINOUS, A. G. \& GI LL, J. M., 1998. The importance of continuity of care in the likelihood of future hospitalization: Is site of care equivalent to a primary clinician? American Journal of Public Health, 88:1539-1541.

MAPELLI, V., 1993. Health needs, demand for health services and expenditure across social groups in Italy: An empirical investigation. Social Science and Medicine, 36:999-1009.

MERZEL, C., 2000. Gender differences in heal th care access indicators in an urban, low-income community. American Journal of Public Health, 90: 909-916.

NEMET, G. F. \& BAILEY, A. J., 2000. Distance and health care utilization among the rural elderly. Social Science and Medicine, 50:1197-1208.

NEWACHECK, P. W., 1988. Access to ambulatory care for poor persons. Health Services Research, 23: 401-419.

NOLAN, B., 1994. General practitioner utilization in Ireland: The role of socioeconomic factors. Social Scienceand Medicine, 38:711-716.

NOVAES, H., 1996. Epidemiology and assessment of health care services: New research trends. Cadernos de Saúde Pública, 12(Sup. 2):7-12.

OAKLEY, A.; RIGSBY, A. S. \& HICKLEY, D., 1993. Women and children last? Class, health and the role of the maternal and child health services. European Journal of Public Health, 3:220-226.

PARKERSON, G. R.; BROADHEAD, W. E. \& TSE, C. K., 1995a. Health status and severity of illness as predictors of outcomes in primary care. Medical Care, 33:53-66

PARKERSON, G. R.; BROADHEAD, W. E. \&TSE, C. K., 1995b. Perceived family stress as a predictor of health-related outcomes. Archives of Family Medicine, 4:253-260.

PERRY, B. \& GESLER, W., 2000. Physical access to primary health care in Andean Bolivia. Social Science and Medicine, 50:1177-1188.

PINHEIRO, R. \& TRAVASSOS, C., 1999. Inequality in health care use by the elderly in three districts in the city of Rio de Janeiro. Cadernos de Saúde Pública, 15:487-496.

ROOS, N. P. \& SHAPIRO, E., 1981. The Manitoba Longitudinal Study on aging: Preliminary findings on health care utilization by the elderly. Medical Care, 9:644-657.

ROSENBACH, M. L.; IRVIN, C. \& COULAM, R. F., 1999. Access for low-income children: Is health insurance enough? Pediatrics, 103:1167-1174.

SALBER, E. J.; GREENE, S. B.; POPE, C. R. \& DAVIS, M A., 1976. Health status, socioeconomic status and utilization of outpatient services for members of a pre-paid group practice. Medical Care, 14:971-986.

SCHOEN, C.; DAVIS, K.; DESROCHES, C.; DONELAN, K. \& BLENDON, R., 2000. Health insurance mar- kets and income inequality: Findings from an international health policy survey. Health Policy, 51:67-85.

SOX, C. M.; SWARTZ, K.; BURSTIN, H. R. \& BRENNAN, T. A., 1998. Insurance or a regular physician: Which is the most powerful predictor of health care? American Journal of Public Health, 88:364370.

SPIVACK, H. R.; LEVY, J. C.; BONNANO, R. A. \& CRACKNELL, M., 1980. Patient and providers factors associated with selected measures of quality of care. Pediatrics, 65:307.

STEIN, A. T., 1998. Acesso a Atendimento Médico Continuado: Uma Estratégia para Reduzir a Utilização de Consultas não Urgentes em Serviços de Emergência. Tese de Doutorado, Porto Alegre: Universidade Federal do Rio Grande do Sul.

SUAREZ, M., 1992. Utilização dos Serviços de Saúde Materno-Infantis na Regi ão Sudoeste da Grande São Paulo. 1989-1990. Dissertação de Mestrado, São Paulo: Universidade Federal de São Paulo.

TITKOW, A., 1983. Illness behavior and action: The patient role. Social Scienceand Medicine, 17:637646.

TRAVASSOS, C.; FERNANDEZ, C. \& PEREZ, M., 1995. Desigualdades sociais, morbidade e consumo de serviços de saúde no Brasil. Série Estudos: Política, Planejamento e Gestão em Saúde, 4:5-26.

VOS, E. T., 1994. Health Seeking Behaviour for Diarrhoea, Acute Respiratory Infections and Malaria. Msc. Thesis, London: London School of Hygiene and Tropical Medicine.

WAN, T. T., 1982. Use of health services by the elderly in low-income communities. Milbank Memorial Fund Quarterly. Health and Society, 60:82-107.

WAN, T. T. H. \& SOIFER, S. J., 1974. Determinants of physician utilization: A causal analysis. Journal of Health and Social Behavior, 15:999-1009.

WASSON, J. H.; SAUVIGNE, A. E. \& MOGIELNICKI, P., 1984. Continuity of outpatient medical care in elderly men: A randomized trial. JAMA, 252:2413.

WOLFE, B. L., 1980. Children's utilization of medical care. Medical Care, 18:1196-207.

WOLINSKI, F. D., 1978. Assessing the effects of predisposing, enabling and illness-morbidity on health services utilization. Journal of Health and Social Behavior, 19:384-396.

YUEN, P. \& BALARAJAN, R., 1989. Unemployment and patterns of consultation with the general practitioner. BMJ, 298:1212-1214.

YUEN, P.; MACHIN, D. \& BALARAJAN, R., 1990. Inequalities in health: Socioeconomic differences in self-reported morbidity. Public Health, 104:6571.

ZAPKA, J. \& AL, E., 1989. Breast cancer screening by mammography: Utilization and associated factors. American Journal of Public Health, 79:14991502.

ZASTOWNY, T. R.; ROUGHMANN, K. J. \& CAFERRATA, G. L., 1989. Patient satisfaction and the use of health services. Explorations in causality. Medical Care, 27:705-723. 